\title{
Imaging and manipulating electrons in a 1D quantum dot with Coulomb blockade microscopy
}

\author{
Jiang Qian ${ }^{1,2}$, Bertrand I. Halperin ${ }^{1}$ and Eric J. Heller ${ }^{1}$ \\ ${ }^{1}$ Physics Department, Harvard University, Cambridge, MA 02138, USA \\ ${ }^{2}$ Arnold Sommerfeld Center for Theoretical Physics and Center for NanoScience, Ludwig-Maximilians-Universität München, Germany
}

(Dated: October 25, 2018)

\begin{abstract}
Motivated by recent experiments by the Westervelt group, which used a mobile tip to probe the electronic state of a segmented nanowire, we calculate shifts in Coulomb blockade peak positions, as a function of tip location, which we term "Coulomb blockade microscopy". We show that if the tip can be brought sufficiently close to the nanowire, one can distinguish a high density electronic liquid state from a Wigner crystal state by microscopy with a weak tip potential. In the opposite limit of a strongly negative tip potential, the potential depletes the electronic density under it and divides the quantum wire into two partitions. There the tip can push individual electrons from one partition to the other, and the Coulomb blockade micrograph can clearly track such transitions. We show that this phenomenon can be used to qualitatively estimate the relative importance of the electron interaction compared to one particle potential and kinetic energies. Finally, we propose that a weak tip Coulomb blockade micrograph focusing on the transition between electron number $N=0$ and $N=1$ states may be used to experimentally map the one-particle potential landscape produced by impurities and inhomogeneities.
\end{abstract}

\section{INTRODUCTION}

Studies of nanoscale electronic structures hold important promise both as laboratories for few-body, interacting quantum mechanical systems and as technological testbeds for future classical or quantum computing technologies. Novel probe technologies 1,2 are very important for studying electronic properties in nanoscale systems because they are often beyond the resolution of conventional imaging techniques like optical microscopy, and traditional transport measurements can only measure spatially averaged physical properties such as the conductance or the current. One scanning probe microscopy $(\mathrm{SPM})^{2}$ technique utilizes a charged metallic tip to perturb the local electronic density in a nanoelectronic structure while monitoring the resulting change in transport properties. Using this technique one can obtain spatially resolved measurement of the electronic properties, including the local electron density and, in principle, the wavefunction itself in the case of a one-electron system ${ }^{3}$ (see discussions below). This imaging technique has been fruitfully applied to study the flow of ballistic electrons across a range of two dimensional heterostructures.

Recently, a series of experiments ${ }^{4}$ applied SPM techniques to study quantum wires. In these experiments a segment of an InAs nanowire lying on top of a two-dimensional $\mathrm{SiO}_{x}$ layer was isolated from the rest of the wire by two short InP segments, forming a one-dimensional quantum dot with lithographically defined boundaries. A negatively charged probe scanned controllably the two dimensional area around the wire and the conductance across the 1D quantum dot was measured as a function of the probe location. Both the voltage of the probe and its height above the surface can also be independently varied. Motivated by these new experimental possibilities, we turn to exact diagonalization techniques to study the conductance response of a few-electron quantum dot as a function of a spatially varied probe potential, in order to illustrate the kind of information that can be extracted in the case of a system of several electrons.

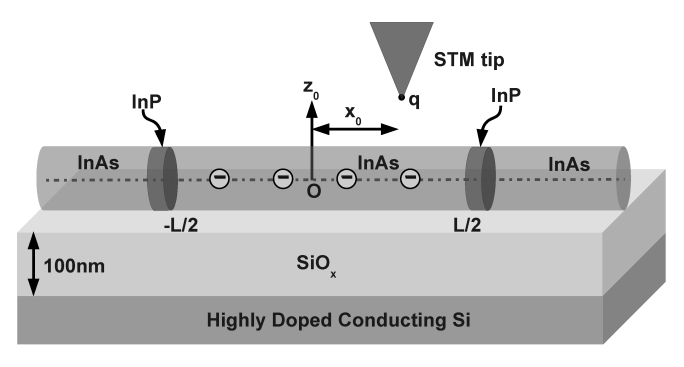

FIG. 1: Schematic geometry of Coulomb blockade microscopy of a quantum wire containing four electrons. When calculating the electron-electron interaction and the electron-tip interaction, we assume that the InP barriers have zero thickness, the InAs wire is infinitely long, and the substrate layers extend to infinity in $\mathrm{x}$ and $\mathrm{y}$ directions.

\section{MODEL}

We consider a uniform InAs (dielectric constant $\varepsilon=15.4$, Bohr radius $a_{B} \approx 34 \mathrm{~nm}$ ) nanowire of radius $R=10 \mathrm{~nm}$, which lies in vacuum atop a $\mathrm{SiO}_{x}(\varepsilon=3.9)$ layer $100 \mathrm{~nm}$ thick, separating it from conducting doped bulk silicon (see Fig. 10. Electrons are modelled as point charges traveling along the center axis of the wire, confined to interval $-\frac{L}{2}<x<\frac{L}{2}$ by hard walls, representing the InP layers. We consider length $L$ from $110 \mathrm{~nm}$ to $500 \mathrm{~nm}$. The electron-electron interaction $U\left(x_{1}-x_{2}\right)$ was calculated using the commercial finite-element program $\mathrm{Comsol}^{\mathrm{TM}}$ to solve the classical Poisson equation for a point charge on the axis of an infinite wire above in a substrate with the geometry described in Fig. 1. At short distance $\Delta x$, the potential was softened to account for the finite thickness of the electron wavefunction, by replacing $\Delta x^{-1}$ with $\left[(\Delta x)^{2}+R^{2}\right]^{-1 / 2}$. Following the approximation used by Topinka ${ }^{5}$, we model the negatively charged probe as a fixed point charge of strength $q$ at a location $\overrightarrow{r_{0}}$ relative to the center point of the wire. This gives rise to a one-body potential $V\left(x ; \overrightarrow{r_{0}}, q\right)$ for an electron on the wire axis at point $x$, which we again obtain by solving the Poisson equation (results are 


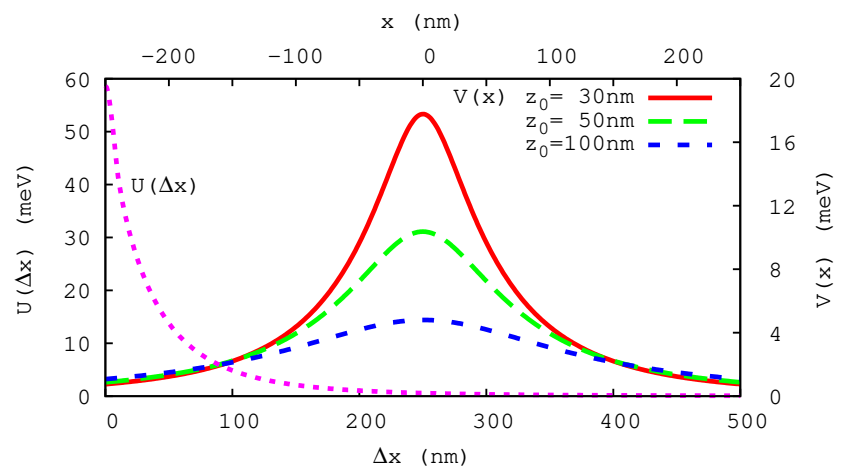

FIG. 2: (Color Online) (Dotted)Interaction potential $U(\Delta z)$.(Solid, long and short dashed) Tip $V(x)$ potentials with a tip charge $q=e$ and locations $\vec{r}_{0}=\left(0,0, z_{0}\right)$ where $z_{0}=30 \mathrm{~nm}, 50 \mathrm{~nm}, 100 \mathrm{~nm}$.

shown in Fig. 2).

In this paper, we diagonalize the exact 1D many-body Hamiltonian with the Lanczos method $\underline{\underline{6}}$ for up to electron number $N=4$ :

$$
-\frac{\hbar^{2}}{2 m^{*}} \nabla^{2} \Psi+\sum_{i=1}^{N} V\left(x_{i} ; \overrightarrow{r_{0}}, q\right) \Psi+\sum_{i=1}^{N} \sum_{j=1}^{i-1} U\left(x_{i}, x_{j}\right) \Psi=E \Psi,
$$

where $\Psi$ is the full many-body wavefunction, depending on the position $x_{i}$ and spin $\sigma_{i}$ of the electrons. To connect to the experimentally observable variables, we consider the Coulomb blockade peak positions of the transition from $(N-1)$ to $N$ electron ground states. The conductance through the quantum wire is maximum when the chemical potential difference between the lead and the wire, controlled by the voltage $V_{g}$ on a back gate, is equal to the ground state energy difference between the two states in question. We may write this condition as $\Delta E \equiv E_{N}-E_{N-1}=\alpha V_{g}+\beta$, where $\beta$ is a constant and $\alpha$ is the proportionality constant between changes in the back gate voltage and the chemical potential in the quantum dot. We probe the electronic states in the quantum wire through the dependence of $\Delta E$ on the tip position $\overrightarrow{r_{0}}$ and potential strength $q$. An interesting set of spatially resolved information about the electrons in the wire can be extracted from this function, and we call this method "Coulomb blockade microscopy". It is a special application of the "scanning probe microscopy" developed by the Westervelt group 2 . In calculations in this paper we focus on the transition from $N=3$ to $N=4$ electrons, but most of our conclusions are easily generalizable to other ground state transitions.

Finally, we note that for four non-interacting electrons with spin in a wire of radius $R=10 \mathrm{~nm}$, when the dot length $L>L_{m}=18.2 \mathrm{~nm}$, the lowest four single particle energy levels are all longitudinal modes. The shortest wire length we consider in this paper $L=110 \mathrm{~nm} \gg L_{m}$, we therefore expect the wires under consideration can be well approximated as strictly 1D under the assumption of weak interaction effects. Indeed, the gap between the transverse ground state and first excited

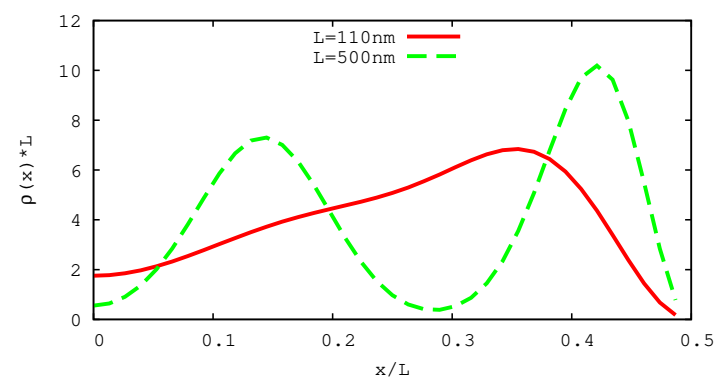

FIG. 3: (Color Online) Electronic densities for a $L=110 \mathrm{~nm}$ wire and for a $L=500 \mathrm{~nm}$ wire in absence of tip potential. Only the right half is shown, as the plot is symmetric about $x=0$. We rescale the $x$ and $\rho(x)$ with wire length $L$ to ease the comparison.

state for our wire is $\Delta E \approx 148 \mathrm{meV}$. The most confined geometry we discuss is shown in Fig. 6, a $L=110 \mathrm{~nm}$ wire with four electrons under an extremely strong tips, squeezing them into the both ends of the wire. Even in that case, the total energy per electron is less than $140 \mathrm{meV}$, not enough to cause an excited transverse mode, with full interaction effects taken into account. Thus we expect the quantum wire to be well approximated by a $1 \mathrm{D}$ model for all the parameters we explored in this paper.

The existence of higher transverse modes will lead to a renormalization of the effective electron-electron interaction (generally a softening of the potential at short distances) even if the energies of the modes are well above the Fermi energy. This is because two electrons that come close together in the lowest transverse mode will mix, virtually, with states where the electrons are in excited modes. As our calculations use, already, a crude phenomenological cut-off at short distances, we do not include explicitly effects of this renormalization. Technically, the existence of higher transverse modes can also lead to three-body and four-body effective interactions, arising from three-body and four-body collisions, but we do not expect such higher body terms to be important in the wires under consideration.

We want to emphasize that although we choose a specific wire geometry and a specific form of interaction that model some characteristics of the setup of the Westervelt group's on-going experiments, most features of the Coulomb blockade micrographs we discuss below are applicable to any $1 \mathrm{D}$ quantum dot system under a mobile potential. Indeed, our discussions of the qualitative features of Coulomb blockade micrographs under both the weak-tip and the strong-tip limits rely only on the general properties of $1 \mathrm{D}$ electronic systems, independent of the specific geometry and interaction we adopt. Our numerical results mostly serve for illustrative purposes. 


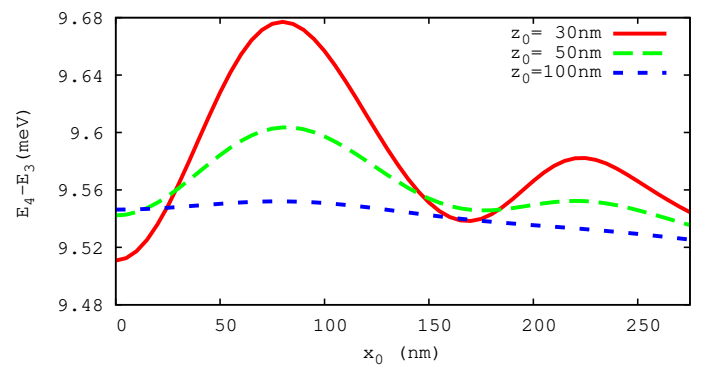

FIG. 4: (Color Online) Coulomb blockade micrographs for a 1D dot with $L=500 \mathrm{~nm}$ and tip charge $q=0.02 e$ for three tip potential shown in Fig 2 Again, the right half is shown.

\section{ELECTRONIC DENSITY}

In the absence of a probe potential $V$, both the $N=3$ and $N=4$ wires the electronic density profile $\rho(x)$ undergoes a crossover as a function of $L$ from a liquid state characterized by a $2 k_{F}$ Friedel oscillations to a quasi-Wigner crystal state characterized by a $4 k_{F}$ density oscillation. Such a crossover from a liquid state to quasi-Wigner states with a decrease in density a very generic phenomenon for 1D interacting fermion system ${ }^{7.8}$. Indeed, for any interacting decaying no faster than $x^{-2}$ at long distance a quasi-Wigner crystal state is known to emerge at low density $\underline{8}$. This requirement for interaction will hold for a system with long screening length as compared to mean inter-particle distance, as is the case for our geometry when the screening doped silicon layer is relatively far $(100 \mu \mathrm{m})$ away. For our specific geometry and interaction, the crossover happens at around density $\rho^{*} \approx 35 \mu \mathrm{m}^{-1}$. A Wigner crystallized density variation is shown in the dashed curve of Fig. 3 for a quantum dot of $L=500 \mathrm{~nm}$, whereas for $L=110$ the four electron density exhibits Friedel oscillations.

\section{WEAK TIP LIMIT}

Now we introduce a weak tip potential, corresponding to a negatively charged tip of strength $q=0.02 e$, scanning above the center axis of the quantum wire along its direction $(1,0,0)$, with the tip location vector $\vec{r}_{0}=\left(x_{0}, 0, z_{0}\right)$. For a $1 \mathrm{D}$ quantum dot of length $L=500 \mathrm{~nm}$, which as shown in Fig. 3 has $4 k_{F}$ Wigner-crystal density variation, let us consider the three tip heights above the quantum wire, $z_{0}=30 \mathrm{~nm}, 50 \mathrm{~nm}, 100 \mathrm{~nm}$, corresponding to the three tip potential shown in Fig. 2. The resulting Coulomb blockade peak position $\Delta E$ as a function of the tip coordinate $x_{0}$ along the wire, i.e. the Coulomb blockade micrograph, is shown in Fig. 4 Clearly, in Fig. 2 the closer the tip approaches the wire, the more localized is the tip potential and a sharper tip potential make it easier to resolve the density variations, this is reflected in Coulomb blockade micrograph scans in Fig. 4. At $z_{0}=30 \mathrm{~nm}, 50 \mathrm{~nm}$ from the tip to the center of the wire, the $4 k_{F}$ density oscillation of the

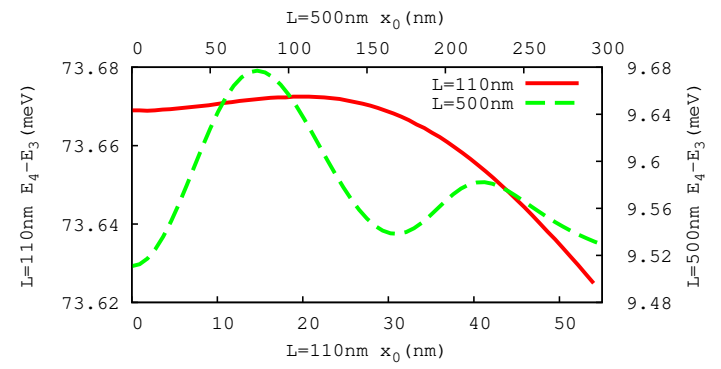

FIG. 5: (Color Online) $q=0.02 e$ weak tip limit for the $N=3$ to $N=4$ Coulomb blockade transition for densities shown in Fig. 3 The tip distance to the wire is $z_{0}=30 \mathrm{~nm}$. Only the right half is shown.

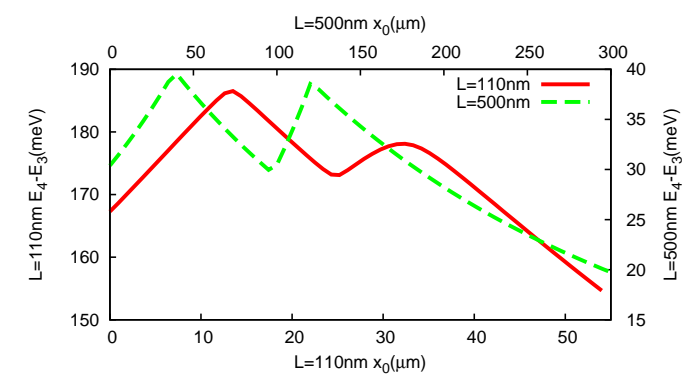

FIG. 6: (Color Online) $q=8 e$ strong tip limit for the $N=3$ to $N=4$ Coulomb blockade transition for densities shown in Fig. 3 The tip to wire distance is $30 \mathrm{~nm}$. Only the right half is scanned.

quasi-Wigner crystal state on the right can be detected in the Coulomb blockade micrograph, whereas when $z_{0}=100 \mathrm{~nm}$ away, the tip potential becomes much too broad to resolve the fine features of the density oscillations. We note that although the resolution of the tip is largely determined by the distance $z_{0}$, the contrast of a Coulomb blockade micrograph, i.e. the magnitude of the $4 k_{F}$ variations in the micrographs, can be improved by modestly increasing the tip potential.

By contrast, in Fig. 5 the $L=110 \mathrm{~nm}$ micrograph at $z_{0}=$ $30 \mathrm{~nm}$ does not show features of Wigner crystal oscillations. However, this micrograph does not by itself give a clearcut indication of the absence of Wigner crystal order for $L=$ $110 \mathrm{~nm}$. With the current interaction and tip parameters, one cannot observe the crossover from the Wigner crystal to the Friedel oscillations because it happens at a inter-particle spacing $\Delta x \approx 30 \mathrm{~nm}$, below the resolution of the micrograph even at $z=30 \mathrm{~nm}$. To be more specific, we may define the onset of Wigner-crystal order for our four-electron system as the length $L$ at which there first appears a local minimum of the mean density $\rho(x)$ in the vicinity of $x / L=0.25$. According to our calculations, this should occur at $\mathrm{L}=135 \mathrm{~nm}$. However, with the tip at height $30 \mathrm{~nm}$, in the weak charge limit, the reso- 


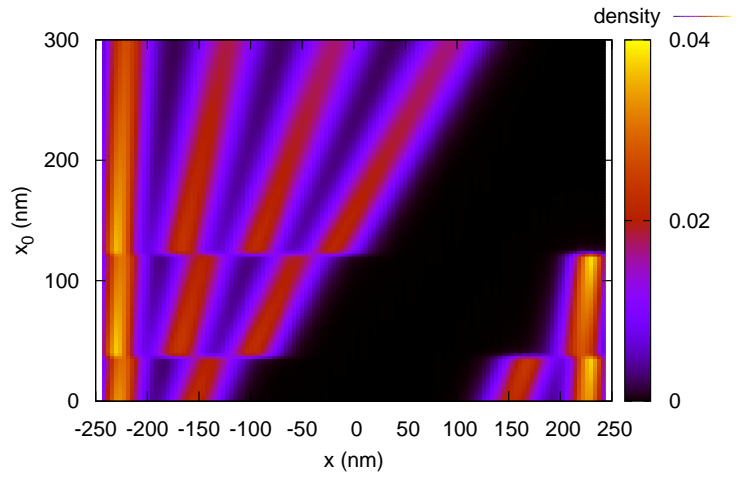

FIG. 7: Electronic densities in $L=500 \mathrm{~nm}, N=4$ quantum wire as a tip $q=8 e$ scan through $0 \leq x_{0} \leq 250 \mathrm{~nm}$. The density throughout the entire wire $-250 \mathrm{~nm} \leq x \leq 250 \mathrm{~nm}$ is shown.

lution of the micrograph is of the order of $60 \mathrm{~nm}$, so we would not see a secondary minimum in the micrograph signal until $L \geq 250 \mathrm{~nm}$.

To gain a more intuitive understanding of a weak tip Coulomb blockade micrograph, we observe that a weak tip only slightly disturbs the electron density as it scans across the wire, thus, a simple first order perturbation theory should be a good approximation to compute the ground state energy in the presence of the tip potential:

$$
E(\vec{r}, q)-E_{0}(\vec{r}, q)=\int d x V\left(\overrightarrow{r_{0}} ; q, x\right) \rho(x),
$$

where $\rho(x)$ is the non-interacting ground state density and $E_{0}\left(\overrightarrow{r_{0}}, q\right)$ is its energy. We have checked that for tip charges up to $q=0.1 e$ the simple first order perturbation theory gives a decent fit to both the ground state energy and the Coulomb blockade micrograph. Since both the width and the center location of the tip potential $V\left(\overrightarrow{r_{0}} ; q, x\right)$ can be adjusted experimentally, the Coulomb blockade microscopy with a weak tip potential provides a flexible way to map the electronic densities in a quantum dot. Indeed, as an example of such flexibility, we find that one can improve the "contrast" of a Coulomb blockade micrograph; i.e., the prominence of the spatial variations in micrographs like Fig. 4 as compared with the total energy shift $E_{4}-E_{3}$, can be improved by slightly increasing the tip potential while still staying within the weak tip perturbative approximation.

\section{STRONG TIP LIMIT}

In the opposite limit of strong tip, the Coulomb blockade tip scans present a very different physical picture. In Fig. 6 we observe that irrespective of whether the electronic state is liquid or Wigner-crystal like as shown in Fig. 3, the Coulomb blockade micrographs show similar behavior: in the case of $N=3$ to $N=4$ transition, both the $L=110 \mathrm{~nm}$ and $L=500 \mathrm{~nm}$
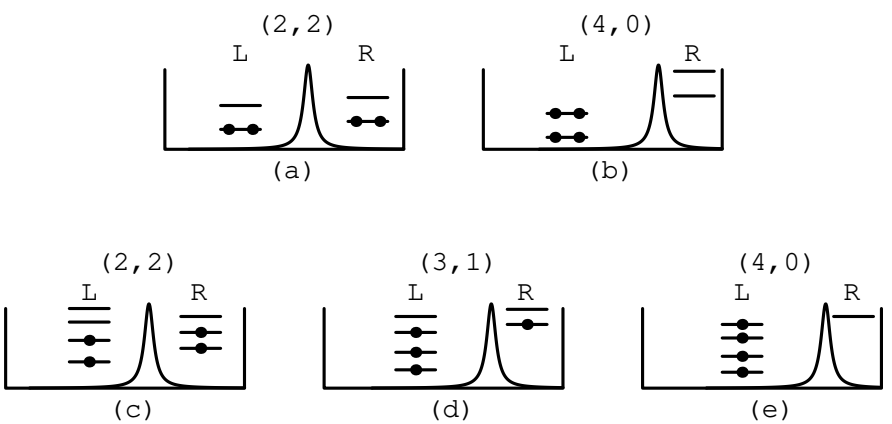

FIG. 8: Schematic illustrations of partition of the four-electron state by the potential barrier under a strong tip potential. In (a) and (b), single-electron energy levels are shown for non-interacting electrons, for two positions of the tip. Because of spin degeneracy, we find either two electrons in each well, or all four in the same well, when the energy of the single-electron ground state on the right becomes higher than the first excited level on the left. In (c)-(e), we "incorporate" interaction energy into "single electron levels" schematically by plotting the energy needed to add an additional electron. In drawing energy levels this way, it is clear that the spin degeneracy in the non-interacting case is lifted by electron interaction, and an additional $(3,1)$ partition will appear for the "energy level" arrangement in (d). Filled circles show electrons in occupied levels.

wire show two relatively sharp peaks for a large tip charge $q=8$. This is in contrast with the case of a weak tip Fig. 5. where the Coulomb blockade micrographs show smooth spatial dependence as well as sensitivity to the electronic states in the absence of the tip potential.

To understand the physics of this strong tip limit we note that the two sharp cusps in Fig. 6 represents discontinuous slope changes in the $N=4$ electron ground state energy as a function of tip position $x_{0}$. Similarly the deep valley in the figure corresponds to a cusp in $N=3$ ground state energy. The origin of these three discontinuities in slopes can be seen in Fig. 7. In this limit, the negatively charged tip potential is so strong that it depletes the electronic density under it. Thus the tip creates an effective partition of the electrons in the wire into left and right sub-quantum dot. As shown in Fig. 7 as the tip move from the center to right of the wire with four electrons, the partitions of the electrons undergoes two abrupt transitions $(2,2) \rightarrow(3,1) \rightarrow(4,0)$. These two transitions correspond to the two upward cusps shown in the $N=4$ curve in Fig. 6 Similarly, the discontinuous slope change shown on the $N=3$ curve of the Fig. 6 corresponds to the transition between the $(2,1) \rightarrow(3,0)$ partition of the ground state. Thus the three discontinuities seen in the Coulomb blockade micrographs in Fig. 6 correspond to, alternately, the transitions between the integer partitioning of total electron numbers in the $N=3$ and $N=4$ system. The upward slope of the curve near $x_{0}=0$ reflects an additional downward cusp at the origin, due to the transition $(1,2) \rightarrow(2,1)$ in the $N=3$ wire.

To better understand the transitions between different partitions, let us consider the transitions in a model of $N=4$ electrons, with spin, which have no Coulomb repulsion between them but interact with a repulsive tip potential. The scenario is illustrated in Fig. 8 , panels $(a)-(b)$. When tip is near the 


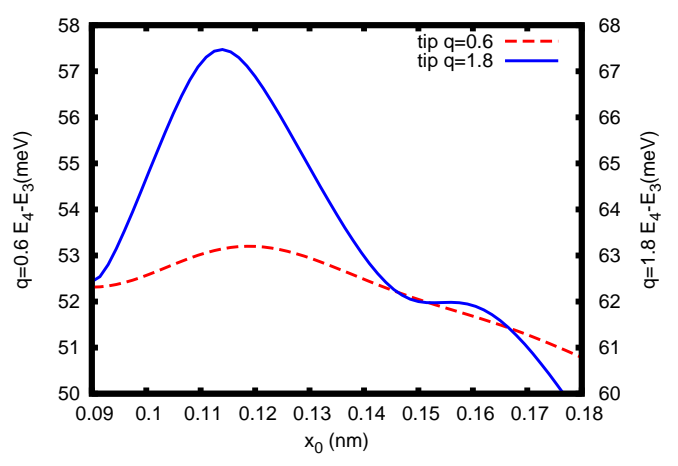

FIG. 9: (Color Online). $q=0.6 e$ and $q=1.8 e$ intermediate tip potentials for $N=3$ to $N=4$ Coulomb blockade transition in a wire of length $L=180 \mathrm{~nm}$. The tip distance to the wire is $z_{0}=30 \mathrm{~nm}$. Only the right half is shown.

center of the wire, the electrons are partitioned $(2,2)$ and both electrons in each side reside in the single-particle ground state. As the tip moves rightward, the energy levels rise in the right partition and fall in the left. When the first excited level on the left partition crosses the ground state on the right, both electrons will move to the left partition. Therefore, contrary to the interacting case, there is no energetically favorable state of $(3,1)$ partitioning in the non-interacting system. In the $N=3$ case, the $(2,1)$ partition is not affected by this, and for noninteracting system the transition $(2,1) \rightarrow(3,0)$ will coincide with the transition in $N=4$, so the Coulomb blockade micrograph will show only a single peak. This analysis can also be generalized to a wire containing multiple non-interacting electrons, such that all the (odd,odd) partitionings of electron number will be missing.

As schematically illustrated in Fig. 8, panels $(c)-(e)$, when we take electron interaction into consideration, the one and two electron state would no longer be degenerate in either partition, so contrary to the non-interacting scenarios in panel $(a)-(b)$, here a $(3,1)$ partition can survive as an intermediate stage between the $(2,2)$ and $(4,0)$ partitions. With the non-interacting case in mind, we postulate that the distance between two peaks in $N=3$ to $N=4$ micrographs, corresponding to the tip positions where $(3,1)$ partitioning in the $N=4$ wire is stable, can serve as an indicator of the relative importance of the interaction energy versus the sum of kinetic and single particle potential energies. The less important interaction is compared to single particle energies, the less splitting would the one and two particle energies be, and the smaller is the region of stable $(3,1)$ partition. This can be seen in Fig. 6. The potential energy should have a larger share in the total energy in the longer wire with lower electronic density, and indeed we observe that the longer wire has a wider distance between the two peaks marking $(2,2) \rightarrow(3,1)$ and $(3,1) \rightarrow(4,0)$ transitions.

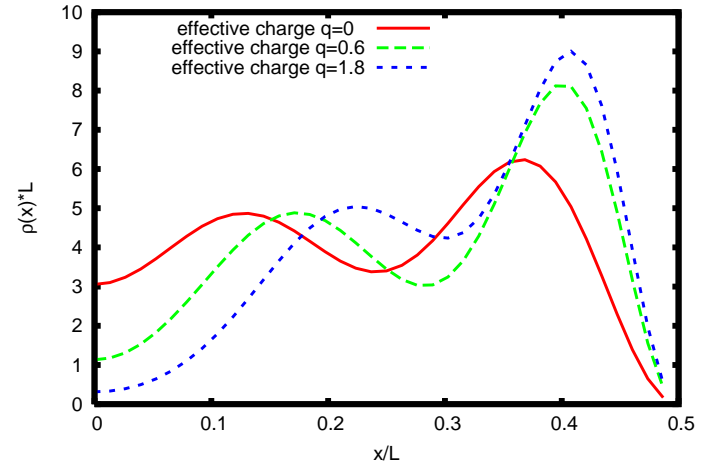

FIG. 10: (Color Online) Electronic density for a $L=180 \mathrm{~nm}$ wire under three tip potentials, with effective charges $q=0 e, q=0.6 e$ and $q=1.8 e$. Only the right half is shown, as the plot is symmetric about $x=0$. We rescale the $x$ and $\rho(x)$ with the wire length $L$.

\section{INTERMEDIATE TIP CHARGE}

We have carried out calculations with various tip charges intermediate between the two limits discussed above. In general, the larger the charge on the tip, the more readily one sees the secondary minima in the micrograph signal, which are seen in Fig. 6 for a charge $q=8 e$, even at $L=110 \mathrm{~nm}$. As one illustration, for a wire of length $180 \mathrm{~nm}$, with a tip height of $30 \mathrm{~nm}$, we find that the micrograph signal shows a secondary minimum at $x / L \approx 0.15$ for $q=1.8 e$, but shows no secondary minimum when $q=0.6 e$, as seen in Fig. 9 However, if we calculate the electron density in the wire when the tip is over the center of the wire $\left(x_{0}=0\right)$, we find that a tip charge of $q=0.6 e$ is enough to substantially modify the density relative to the density in the absence of the tip. As seen in Fig. 10, the electron density below the tip, at $x=0$ is reduced by a factor of three relative to the density with no tip charge. Nevertheless, the oscillations seen in $\rho(x)$ remain qualitatively similar to structure seen in the absence of the tip. For example, the charged tip only pushes out the position of the secondary minimum in the density from $x / L=0.25$ to $x / L=0.3$.

In general, when we increase the tip potential to intermediate values, the resolution of our micrographs improves compared with the weak tip limit. This is evident in the fact that at $q=1.8 e$ we can already see signs of Wigner crystallization at $L=180 \mathrm{~nm}$, in contrast with the weak tip case with $q=0.02 e$ where we can only detect quasi-Wigner crystal at $L=250 \mathrm{~nm}$. On the other hand, at $q=1.8 e$ there is no signature of Wigner crystallization when the electrons are in a liquid state in a $L=110 \mathrm{~nm}$ wire. Thus we do not have "false positive" signature of quasi-Wigner crystal, in contrast with the strong tip limit described in Section $\nabla$ where the micrographs show $N$ peaks for a $N$-electron wire regardless whether, in the wire in the absence of the tip, the electrons are in a liquid or a quasiWigner crystal state.

Because of these two characteristics, an intermediate tip potential may help an experimentalist to reliably detect the presence of a quasi-Wigner crystal state in a shorter, higher density 
wire closer to the crossover from a liquid state.

\section{SINGLE ELECTRON}

Beyond the system of interacting electrons discussed above, a possible further application of Coulomb blockade microscopy is to experimentally "map" the rugged potential landscape produced by wire inhomogeneities and charged impurities in the substrate. One would focus on the transition from $N=0$ to $N=1$ state, in which case the Coulomb blockade micrograph would reveal information about the single particle density. By inverting the transformation in Eq. 2, one may be able to approximately obtain the single particle ground state density $\rho(x)$. In the absence of an external magnetic field, the ground state wavefunction $\psi(x)$ has no nodes and can be chosen to be $\psi(x)=(\rho(x))^{1 / 2}$ It is then straightforward to invert the Schrödinger's equation to extract the potential landscape from the single particle wavefunction.

\section{SUMMARY}

In summary, in this paper we show that tracking the peak position shift as a charged mobile tip moves above and across a nanowire, a technique we term Coulomb blockade microscopy, can reveal spatially-resolved information about the electronic density and states of a quantum 1D dot. A weak tip potential can serve as a probe with a tunable width, to reveal the spatial distribution of the electronic density in the wire. A strong tip potential that depletes part of the wire can be used to manipulate individual electrons from one partition to the other, and the accompanying Coulomb blockade micrograph can indicate the transitions between different partitionings. Furthermore, a feature of the resulting micrograph, the distance between peaks marking the (odd,odd) partitioning, can serve as an indicator of the relative strength of the interaction.

In this paper we have chosen extreme values of the tip charge $q$ to illustrate the physics in the two limits. However, our calculations show that the discussions above hold true for a wider range of moderately small and large values of $q$.

To obtain a quantitative description of the energy shifts expected in Coulomb blockade microscopy, particularly in the intermediate coupling regime, we see that it is necessary to perform a realistic calculation, which takes into account both the electron-electron interaction and the non-linear effects of the charged tip on the electronic state of the wire. If one is prepared to carry out such a calculation, however, Coulomb blockade microscopy can be a powerful probe of interaction effects in the wire.

\section{ACKNOWLEDGEMENTS}

We would like to thank Erin Boyd, Halvar Trodahl and Jesse Berezovsky and especially Bob Westervelt for helpful discussions. This work is supported in part by NSF grants PHY-0646094 and DMR-0906475. Numerical work was performed in part at the Center for Nanoscale Systems, a member of the National Nanotechnology Infrastructure Network (NNIN) supported by NSF award ECS-0335765. JQ is also supported in part by NIM and DFG through SFB 631.
1 O. M. Auslaender, A. Yacoby, R. de Picciotto, K. W. Baldwin, L. N. Pfeiffer and K. W. West Science, 295(5556):825-828, 2002.

2 M. A. Topinka, R. M. Westervelt and E. J. Heller Physics Today, 56(47), 2003 and refs. therein.

3 P. Fallahi, A. C. Bleszynski-Jayich, R. M. Westervelt, J. Huang, J. D. Walls, E. J. Heller, M. Hanson and A. C. Gossard, Proc. 27th Int. Conf. on Phys. of Semiconductor, AIP, 2005, p. 779.

4 A. C. Bleszynski-Jayich, L. E. Fröberg, M. T. Björk, H. J. Trodahl, Lars Samuelson and R. M. Westervelt, Phys. Rev. B, 77, 245327
2008.

5 M. A. Topinka. PhD thesis, Harvard University, 2002.

6 J. K. Cullum and R. A. Willoughby. Lanczos Method for Large Symmetric Eigenvalue Computation, Vol. 1. Birkhauser Boston Inc., Boston, MA, 1985.

7 G. A. Fiete, J. Qian, Y. Tserkovnyak, and B. I. Halperin Phys. Rev. $B, 72,045315,2005$.

8 K. A. Matveev, Phys. Rev. B, 70, 245319, 2004. 